\title{
Bone Age Measurement using a Hybrid HCNN-KNN Model: A Case Study on Dental Panoramic Images
}

\author{
Fatemeh Sharifonnasabi ${ }^{1}$, NZ Jhanjhi ${ }^{2}$, Shahab Shamshirband* ${ }^{3}$, Jacob John ${ }^{4}$, Hamid \\ Alinejad-Rokny* 5,6,7 \\ 1,2 School of Computing \& IT (SoCIT), Taylor's University Subang Jaya, Malaysia. \\ Fatemehsharifonnasabi24@gmail.com, noorzaman.jhanjhi@taylors.edu.my \\ ${ }^{3}$ Future Technology Research Centre, College of Future, National Yunlin University of Science and \\ Technology, 123 University Road, Yunlin 64002, Taiwan. shamshirbands@yuntech.edu.tw \\ ${ }^{4}$ Department of Restorative Dentistry, Faculty of Dentistry, University of Malaya, Kuala Lumpur, \\ Malaysia.drjacob@um.edu.my \\ ${ }^{5}$ BioMedical Machine Learning Lab (BML), The Graduate School of Biomedical Engineering, \\ UNSW SYDNEY, Sydney, 2052, AUSTRALIA. h.alinejad@unsw.edu.au \\ ${ }^{6}$ Core Member of UNSW Data Science Hub, The University of New South Wales (UNSW \\ SYDNEY), Sydney, 2052, AUSTRALIA. \\ ${ }^{7}$ Health Data Analytics Program Leader, AI-enabled Processes (AIP) Research Centre, Macquarie \\ University, Sydney, 2109, AUSTRALIA.
}

To whom correspondence should be addressed. Tel: +61 29385 3911; e-mail:

shamshirbands@yuntech.edu.tw and h.alinejad@unsw.edu.au

\begin{abstract}
Bone age measurement is the process of evaluating the level of skeletal maturity to estimate actual age of bone. This evaluation is usually done by comparing a radiograph of a bone with an existing standard chart that includes a set of identifiable images at each stage of development. Manual methods are based on the analysis of specific areas of bone images or dental structures. Both methods are highly dependent to human experience and are timeconsuming. An automated model therefore is needed to estimate the age accurately. In this study, we propose a hybrid convolutional neural network (CNN) combining $\mathrm{K}$ nearest neighbours (KNN) and PCA to estimate the age of bone automatically and accurately. We applied our model, HCNN-KNN, on a dataset collected by dental teaching institutes and private dental clinics in Malaysia. A total of 1,922 panoramic dental radiographs of dental patients aged between 15 to 25 years old were obtained from the various centres. These radiographs were separated by age, classified as those in the range of 12-months, six-months, three-months, and one-month gaps. This novel investigation, implemented for the first time with precision to the range of the age for \pm twelve months, \pm six months, \pm three months, and \pm one month, and these age ranges determine the age of minors which could help the model to find better features and train the model more accurately. Replacing SoftMax with KNN generally improves traditional CNN performance to reduce the noises in images. Therefore, the optimal number of image similarities in a larger dataset is more significant, and the proposed method can benefit
\end{abstract}


1 from large amounts of annotated data. Since the similarities of radiographic images are very similar, there may be several similar possibilities in the SoftMax classification method. These similar probabilities increase the risk of misdiagnosis of bone age measurements. Therefore, replacing KNN with SoftMax is the best choice for age group differentiation in classifiers. Finally, the accuracy rate is evaluated with the accuracy criterion according to the equation in confusion metrics and comparing existing models. The accuracy results on the dataset by \pm 12 months, \pm 6-months, \pm 3-months, and \pm 1-month are 99.98, 99.96, 99.87, and 98.78, respectively.

Keywords: Dental Age Measurement, Dental Radiography, Orthopantomogram, Convolutional Neural Network, K-Nearest Neighbour, Health Data Analytics, Biomedical Machine Learning.

\section{Introduction}

Determining a person's actual age is legally essential, and the accurate identification of an unidentified deceased is presently still a primary forensic concern. A solid reason to identify the exact age of unknown individuals with a precise algorithm to solve the issue is at present. Many illegal immigrants have died from natural disasters or the Coronavirus (COVID-19), which is killing thousands of people around the world (Amon, 2020; Clark et al., 2020; Satterstrom et al., 2020). Assessing the age of unknown human corpses is significant in determining criminal investigations or a collective catastrophe because the age at death, date of birth, year of death, as well as gender, can guide researchers toward the correct identity among many matching cases (Sironi et al., 2018; Kapadia, Stevens, \& Silver, 2020).

Most previous approaches for age estimation are in the primary stage in terms of accuracy. Skeletal age can be measured using several different human bones (Wik et al., 2020; McGill, 2021). The high cost, long-term monitoring, and risk of radiation exposure suggest that this is not feasible or practical for maximum human body segments (Shi et al., 2020).Researchers have looked at the entire progress of the frame and examined the numerous approaches used in different regions of the body (Lee et al., 2017; Spampinato, 2017; Shobha Rani, 2021). Extensive medical age measurement methods are usually extracted based on centers' body parts (Mutasa, 2018). The regions used to measure bone agefrom the body comprise the foot, shoulder, ankle, hip, elbow, cervix, ankle, and teeth (Dahlberg, 2019). Therefore, maximum age estimation used hand wrist and face characteristics (McGill, 2021; Shen, 2021). However, the accuracy of these sections in estimating age may be affected by age, race, genes, work environment, lifestyle, smoking, health status, and even other factors such as mortality and disaster (Gurpinar, 2016).

To achieve the best and most accurate age detection from radiographic images, the use of dental panoramic images is more effective than comparing to other parts of the body images as teeth are immortal, long-lasting, and will even remain in high temperature and a dead body (Ezhil, 2018). Institute of Forensic Medicine claimed that the X-ray images of teeth are the primary and most used method in estimating the dental age (Čular, 2017; Houssein, 2020). 
1 As stated above, the skeletal maturity index of the extraction centre radiographs is bone age (Cameriere, 2019). Despite the great content of scientific research on dental age measurement, there is no consensus on the validity of bone age measurement (BAM) methods adapted for use in clinical ambience (Bagattoni, 2019). In childhood, dental age can be correctly assessed because most of the teeth are evolving concurrently (Ginnis, 2019). Still, among minors and the adolescent population, age measurement cannot be precise (Asif, 2019) unless a predictive model with optimal layers is provided for accurate results, which has not yet been developed (Čular, 2017).

Traditional dental age measurement is a manual method that may include several steps such as segmentation, feature extraction, image pre-processing, classification, or regression. The form and variations in the above stages are seen in dry bone and radiographer images. Age nomination is undoubtedly based on the timing of the emergence of extraction centres, identifying the region of epiphysis and fusion, depending on whether the bone is dry, or using an imaging technique such as radiography. Age segmentation assessment is based on a corresponding procedure that involves comparing a radiographer's image of the subject to an existing reference that includes a known gender and age sample. Estimating age is mainly a measurement of biological maturation, which is changed into a time by comparing a picture with a specified reference (De Tobel, 2020). A clear disadvantage of this traditional bone age method is the accuracy in determining the age, especially in determining young age (minors age) because this method uses to identify the age of the person by comparing standard charts of the region of bone growth which usually cannot be an accurate as the bone growth changes in a one-person depend on the health individual's care and lifestyle. An inversion in these strategies, considering the differences in anatomical structure, the range of accuracy is very wide, and the results came with the problem of low accuracy that leads this research to use machine learning techniques instead of conventional methods.

Research has shown that most researchers have used machine learning algorithms like NN, SVM, KNN, Decision Tree in bone age measurement (Tao et al., 2018; Farhadian, 2019; Hemalatha., 2021). Machine learning algorithms cannot process data in raw form, as they are subject to errors that lead to inaccurate class recognition. Therefore, carefully engineered attribute extractors need to convert raw data into attribute vectors for proper classification. However, extracting characteristics that can accurately represent behaviour in different environmental conditions is a challenging task that has left a wide range of research open to researchers in thisfield (Houssein, 2020; Spampinato, 2017). Hence, choosing a classifier that can differentiate behaviours in terms of diversity within and outside the classroom is one of the challenges in recognizing behaviour (Kalantari, 2018; Shamshirband, 2019; Shamshirband, 2020). Moreover, deep learning approaches are effectively used to resolve several concerns in many analysis areas and computer vision.

Most researchers have used machine learning algorithms like NN, SVM, KNN, Decision Tree in bone age measurement (Tao et al., 2018; Farhadian, 2019; B. Hemalatha, Rajkumar, N., 2021). However, Machine learning algorithms cannot process data in raw form, as they are subject to errors that lead to inaccurate class recognition. Consequently, carefully engineered 
1 attribute extractors need to convert raw data into attribute vectors for proper classification.

2 However, extracting characteristics that can accurately represent behaviour in different 3 environmental conditions is a challenging task that has left a wide range of research open to

4

5

6 researchers in this field (Spampinato, 2017; Houssein, 2020). Therefore, choosing a classifier that can differentiate behaviours in terms of diversity within and outside the classroom is one of the challenges in recognizing behaviour (Kalantari, 2018; Shamshirband, 2019; Shamshirband, 2020). Moreover, deep learning approaches are effectively used to resolve several issues in many analysis areas and computer vision.

Deep learning-based methods, also known as end-to-end learning-based methodologies, are those in which deep neural networks, such as convolutional neural networks, operate directly on the input images and generate the desired output without intermediate steps such as segmentation and extraction (Haque, 2020). However, developing and training deep neural networks can be a challenging and time-consuming process. It is possible to use a pre-trained deep network to perform the desired tasks rather than creating and teaching deep neural networks from scratch. Transfer learning is a term used to describe this process (Qummar, 2019; Li, 2020).

Today, machine learning techniques has been used widely to identify patterns in complex data including medical data. i.e., genomics, bioimaging, and phenotypic data [Alinejad-Rokny, 2016; Parvin, 2011; Javanmard, 2013; Ahmadinia, 2013; Mahmoudi, 2021; Hosseinpoor, 2020]. In this article, a novel automated machine learning model is proposed for bone age measurement based on dental panoramic images using deep convolutional neural network techniques to improve the accuracy of determining age in less than one year (+/- six months) (Sharifonnasabi, 2020). The proposed method determines to obtain the precise age between 9 groups of age in the range of 15 to 23 . These age groups are included in year classes themselves which are six months, three months, and one month's classes, with a combination of the methodology of convolutional neural networks and (as extraction and analysis of information and features in dental images) the nearest neighbour (as a classification method).

Figure 1 shows the architecture of the proposed HCNN-KNN model used in this article. The architecture of this model consists of four steps. The collected clinical data of X-ray OPG is the input images as the first step. In the second step, the model will be trained by a convolutional neural network (CNN). In the third step, the fully connected layer from CNN will be used as an input for PCA to perform data transform and dimensionality reduction. In the last step, this data is classified using the KNN algorithm to predict classes in the dataset, explained in detail in Section Three, the proposed model. 


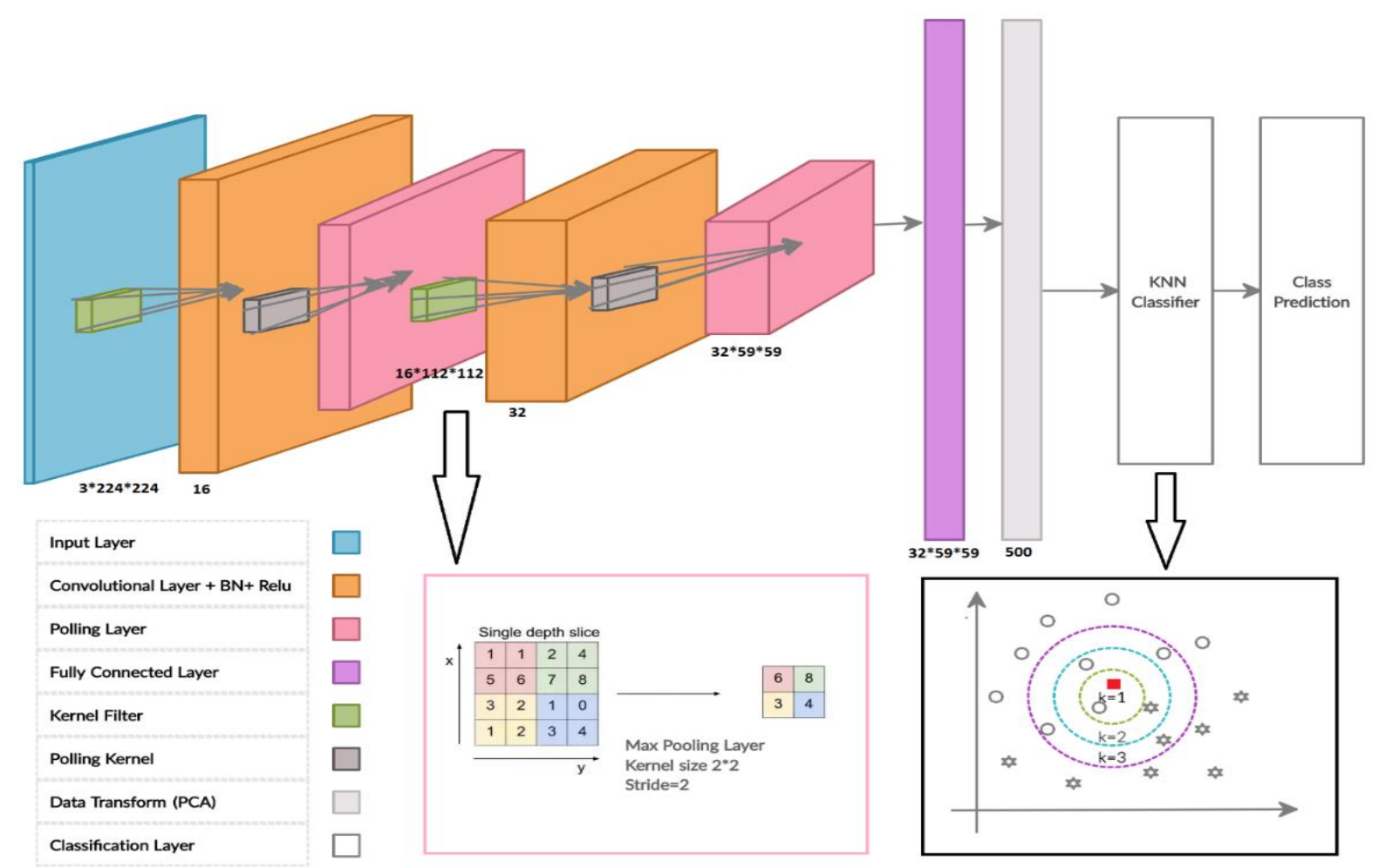

Figure 1. HCNN-KNN Hybrid Model Proposed Architecture Based on Dental X-Ray OPG Images

2 This paper is divided into five sections: The strategies for determining dental age are detailed in Section 2. The different phases of the proposed automated dental age calculation are depicted in Section 3. The deployment elements and performance review are covered in Section 4. As a result, Section 5 is divided into two sections: conclusion and future work.

\section{Related Literature}

Several approaches have been applied to identify the age using dental images. A deep neural network algorithm estimating the age using dental X-ray images was used as a foundational forensic science role. The features are derived using two deep neural networks, AlexNet and ResNet, in the recommended process. A dataset with 1,429 dental X-ray images was used. The proposed approach is tested using several acceptable output parameters (Houssein, 2020).

Avuçlu et al., 2020 used a morphological measurement on dental X-ray images 2020 to assess age and gender. Details of age and gender were typically identified by function and dental Xray images of the teeth. With 1315 dental images and 162 different dental classes, the photos went beyond the borders. This dental knowledge is also contained in separate XML directories. The highest estimated age and gender estimates are 95\% for accuracy (Avuçlu, Başçiftçi, Fatih, 2020).

Farhadian et al., (2019) introduced a ground-breaking prediction system. They used a neural network that relied on dental data to alter age assessment. The age group of between 14 and 60 was used, and the overall data sample size for the research was three hundred images. For 
statistical analysis, SPSS21 statistical software and R were utilized. The neural network methodology, with an MAE of 4.12 years, showed reasonably good results (Farhadian, 2019). The neural network errors were smaller than the regression model's estimation errors, with the test data set being RMSE of 10.26 years and MAE of 8.17 years.

Alkaabi et al. (2019) examined different Convolutional Neural Network architectures for age estimation. The tests utilize CNN to fix the inconvenience of forensic dentistry's automatic age calculation without any modifications. The transfer learning principle is also used to train the common CNN architectures for age estimation, such as AlexNet, VGGNet, and ResNet. Age estimation efficiency is measured by evaluating its recall, accuracy, F1-score, accuracy, and average accuracy for all the tested architectures. It has been claimed that this is the first paper that seeks to predict age estimation from dental images using Capsule-Net with the best of knowledge. However, the proposed architecture indicates that Capsule Network has increased $36 \%$ over CNNs and transfer learning to reach a cumulative accuracy of $76 \%$ (+_ 1 Year).

Tao et al. (2019) has introduced the Multilayer Perceptron Neural network to estimate dental age. The experiments are carried out on a dataset composed of 1636 samples. It was also experimentally confirmed that this latest feature set makes the dental age estimation more reliable $\left(+\_\right.$Year $)$.

An age evaluation approach was tested on Malaysian adolescents between the ages of 1 to 17 . The first to the third molar teeth were segregated first. Next, based on an intensity projection method, the invariant deformation characteristics were collected. Finally, the designed DCNN model extracts a broad range of features in the hierarchical layers: invariance of size, rotation, and deformation. Using a systematic and branded orthopantomography of 456 patients recorded by the Department of Dentistry and Study of University Sains Islam Malaysia, the process using this technique was then evaluated (+_1 Year).

Kim et al. (2019) applied deep learning algorithms to estimate age using dental panoramic images and stated that this assessment is a fundamental task in forensic science. The dataset used incorporates dental X-ray pictures of 9435 people (4963 male, 4472 female) sorted out in three-age gatherings. The result of deep learning algorithms based on CNN neural networks shows that the proposed approach functions evaluated based on a database of panoramic dental radiographs and worked well for accuracy.

Banar et al. (2020) implemented a fully automatic staging method leveraging the total capacity of deep learning in every stage of the system, using convolutional neural networks. The dataset used 400 panoramic radiographs with 20 OPGs per stage of development per gender, arranged with the agreement of three observers. In dealing with a restricted dataset, transfer learning principles, using pre-trained CNNs, and data augmentation was used to minimize the problems. On average, it took $2.72 \mathrm{~s}$ for the entire automatic workflow to compute, which is considerably quicker than conventional staging starting from the OPG. Given the small size of the dataset, this pilot study indicates that the completely automated solution suggested demonstrates encouraging outcomes relative to manual staging. 
1 A semi-supervised fuzzy clustering algorithm with spatial limitations for dental segmentation

2

3

4

5

6

7

8

9

10

11

12 from X-ray images was introduced by Tuan et al. (2017). The experimental findings show that the recommended work has superior accuracy than the initial semi-supervised fuzzy clustering and several related approaches.

De Tobel J. (2017) developed machine learning algorithms to evaluate the growth of bones of the hands, wrists, and dental on radiography and magnetic resonance imaging. Twenty panoramic radiographs at every step for each sex were selected for the World Dental Federation notation element (FDI) 38. The two monitors were unanimously determined on the steps. If needed, a third viewer functioned as the referee to collect the third molar reference point. The radiographic collection was used as training data. Initially, image optimization was the contrast setting for evaluating the third molar, and a standard rectangular box was positioned all around it is using Adobe Photoshop CC 2017. This narrow box shows the area for the next step. Then, the machine learning algorithms are utilized to find the automatic phase. The classification presentation was then measured in a 5-fold reliability situation using several credibility criteria (accuracy, degree-N detection speed, mean absolute difference, kappa linear coefficient). The mean accuracy was 51.0, the average unconditional disagreement was 0.6, and the kappa average was 0.82 in $\left(+\_1\right.$ Year).

Age of majority is the threshold of adulthood that is recognized or declared in the law. Most countries set the age of the majority at 18 . Still, some jurisdictions are older, and others are younger. The word majority in this context refers to older years and having a full age versus a minority, at is, being a minor. When minors are considered as an adult, the legal control and responsibilities of their parents or guardians over them will end (Sharifonnasabi, 2020). Minors may be legally exempted from certain privileges such as voting, buying, and drinking alcohol, buying tobacco or cannabis products, gambling, marriage, buying or owning a firearm, owning property, or getting married, migration, adult sports, or get full driving privileges (Kruessmann, 2021; Sharifonnasabi, 2020).

Considering the multiple related issues in the age of majority, which is the part of essential matter in legal and forensic and sports organizations, estimating age in forensic medicine and clinical dentistry is particularly important. Age estimation based on tooth improvement is a consistent method as tooth bones are immortals, and 18 to 20 years, the only skeletal components visible in the macroscopic of these facial bones are teeth through post-mortem decomposition (Blatt, 2020). As a result, the tooth and jawbone are selected to develop the hybrid method because it is a helpful bone for age measurement amongst parts of the forensic association. It becomes essential that a predictive model to measure bone age is identified. Modelling an accurate method for measuring age in the medical field can help accelerate personal identification and thus reduce costs. Although the number of bone age measurement (BAM) models has increased, most remain in the testing phase because precise results have not been obtained, especially in predicting the minor's age. Furthermore, there are no robust computerized methods for BAM within the healthcare setting, with a precision to the range of +_ six months for dental age estimation techniques due to image analysis and image processing techniques limitations (Sharifonnasabi, 2020). 


\section{The proposed model}

2 In this section, the generation of the HCNN-KKN hybrid model, the conceptual framework of 3 image processing and the dental classification process are in five steps is described. Data 4 collection, Pre-processing, Modelling, evaluation, and validation are the five steps of this 5 process which are explained in detail following Figure 2.

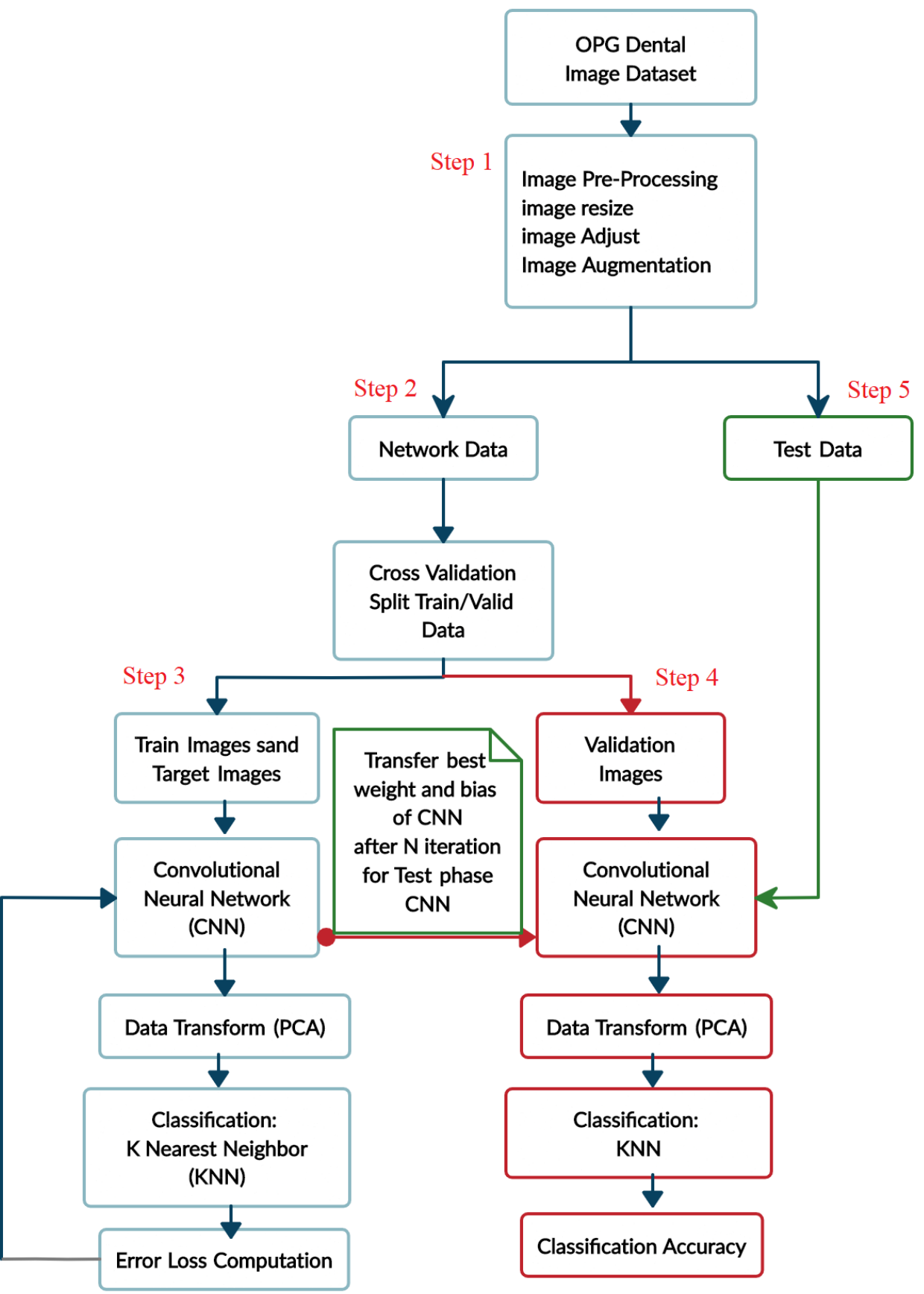

\section{Step 1: Image Pre-Processing and Data Augmentation}

9 For the better resolution of OPG x-ray images, a series of pre-processing and normalization 10 operations are used. Initially, the images were removed from the extra margins and cropped. 11 The image is then adjusted to increase the contrast of the images and highlight the images' 
1 edges. It is also done to normalize the image with zero base and variance 1. A lot of data is also

needed from OPG $\mathrm{x}$-ray images to train CNN better. If numerous images are not available, data augmentation is used. In this study, data mirroring and data duplication have been used to increase the number of samples.

\section{Step 2: Split Dataset to Train, Valid and Test Dataset as Cross-Validation Step}

To perform the classification, the data in the dataset are divided into two categories: train data and validation data. This classification has been done in the current research, including crossvalidation with 50-50, 70-30, and 20-80. For example, 20-80 means that $20 \%$ of the dataset data is validated for validation and $80 \%$ of the dataset data for the train. But for test operations, new data collection with special features has been used. This data is new and was not a part of out training and validation processes, and the data is a combination of X-Ray OPG images of different races, with different genders and at different ages. Obtaining acceptable results on various test data will indicate the correct performance of the proposed model.

\section{Step 3: Hybrid HCNN-KNN}

The architecture of the proposed HCNN-KNN model is considered in this research according to Figure 1. In this step, the architecture used in this methodology includes an inputs layer, 2 Convolutional layers, a fully connected layer, PCA transform data layer, and a KNN layer for classification. The first layer of this model is an input image of a dimension with zero centre normalization and a size of $224 * 224$ pixels. These input images are in RGB colour. The convolutional layer is the second layer in this model, and it contains 16 convolutional filters with a kernel size of 5 and 2 paddings. Low-level features like edges, blobs, shapes, etc., are obtained in this stage. A batch normalization in this layer is 16 and a nonlinear activation function, ReLU. This convolutional layer is named 'conv1'. The Maxpooling layer downsamples the model with stride 2 and 2 paddings.

As a result, after the Maxpooling layer, the dimensions of the images in this layer are reduced to $112 \times 112$. Therefore, considering the number of 16 convolutional layer filters in conv 1 and after the pooling operation, the output of this layer will be equal to $112 \times 112 \times 16$. The proposed model has two such convolutional blocks. The second convolutional block is similar to the first convolutional block, but the convolutional layer has 32 filters with a kernel size of 5 and 2 paddings.

Similarly, to the conv1 layer, the output of conv 2 will be $56 \times 56 \times 32$. Then fully connected layer is used to connect all the neurons in the layers. Therefore, Table 1 shows the parameters used in the $\mathrm{CNN}$ network. In the $\mathrm{CNN}$ model, 50 percentage dropout layers are connected between fully connected layers to reduce the features to the next layer. In this Classification problem, the number of classes is 9 , like 15 to 23 years (number of class 18 like the first six months of 15 years, the second six months of 15 years to The first six months of 15 years, the second six months of 15 years ( \pm six months); the number of class 36 like the First trimester, second trimester, third trimester and fourth trimester, from 15 years to the First trimester, second trimester, third trimester and fourth trimester, from 23 years ( \pm three months); the number of classes 108 like every 12 months or 15 years to every 12 months of 23 years ( \pm one month). So that the last fully connected layer is modified as nine classification tasks with 
1 parameters like bias learn rate factor and weight learn rate factor. But in our proposed HCNN-

2 KNN model.

3 Table 1: A Display of the Parameters Used in The CNN Network

\begin{tabular}{llccccc}
\hline & type & $\begin{array}{l}\text { Filter } \\
\text { size }\end{array}$ & stride & \# Filters & FC & Input \\
\hline Layer1 & Convolution & $5 \times 5$ & $2 \times 2$ & 16 & - & $224 \times 224 \times 3$ \\
Layer2 & Maxpooling & $2 \times 2$ & $2 \times 2$ & - & - & $224 \times 224 \times 16$ \\
Layer3 & Convolution & $5 \times 5$ & $2 \times 2$ & 32 & - & $112 \times 112 \times 16$ \\
Layer4 & Maxpooling & $2 \times 2$ & $2 \times 2$ & - & - & $112 \times 112 \times 32$ \\
Layer5 & Fully connected & - & - & - & $*$ & $59 \times 59 \times 32$ \\
\hline
\end{tabular}

*9 for Dataset on Year, 18 for a dataset on \pm 6 -months, 36 for a dataset on \pm 3 months, and 108 for a dataset on \pm 1 month

4

In this step, instead of the SoftMax probability layer, the combined CNN model with KNN is used as a classifier to evaluate the class event. But at this stage, before the fully connected layer data enters the KNN layer, we propose to transfer the data to space with more distinct information and smaller dimensions using the principal component analysis method and call it 'Fully_PCA'. Therefore, fully connected layer neurons after PCA data transformation and reduction are considered overlays of the KNN classification layer. In this case, the KNN input is assumed to be 500 .

Figure 3 shows the HCNN-KNN model algorithm pseudo-code proposed in the bone age measurement problem based on dental images. $\mathrm{K}$ are 1, 2, 3 in $\mathrm{KNN}$, and the distance metric is Euclidean distance. The model is trained using an SGD momentum optimizer with a 0.0001 learning rate. The model is then trained for ten epochs with a mini-batch size of 16 images. Finally, after learning the network and classification operations, the error loss rate is obtained to evaluate the proposed network model in subsequent epochs of network learning.

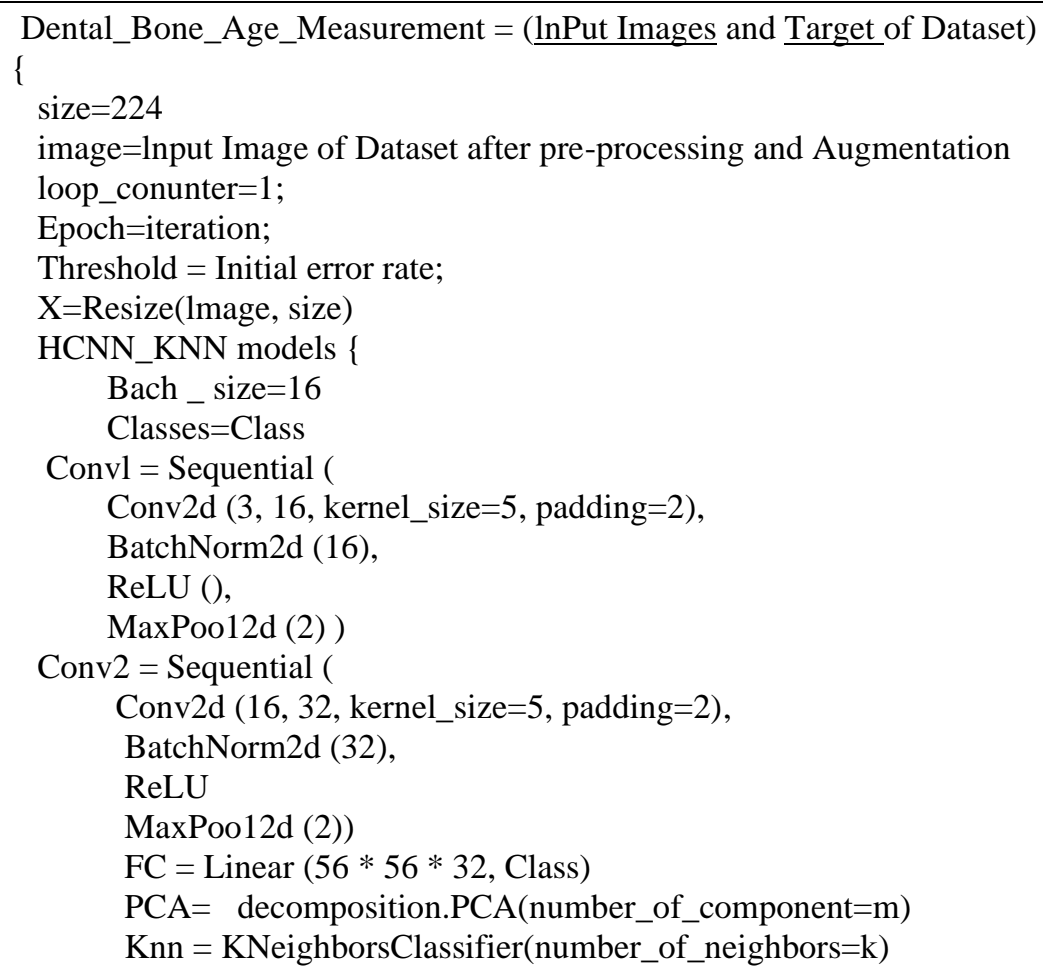




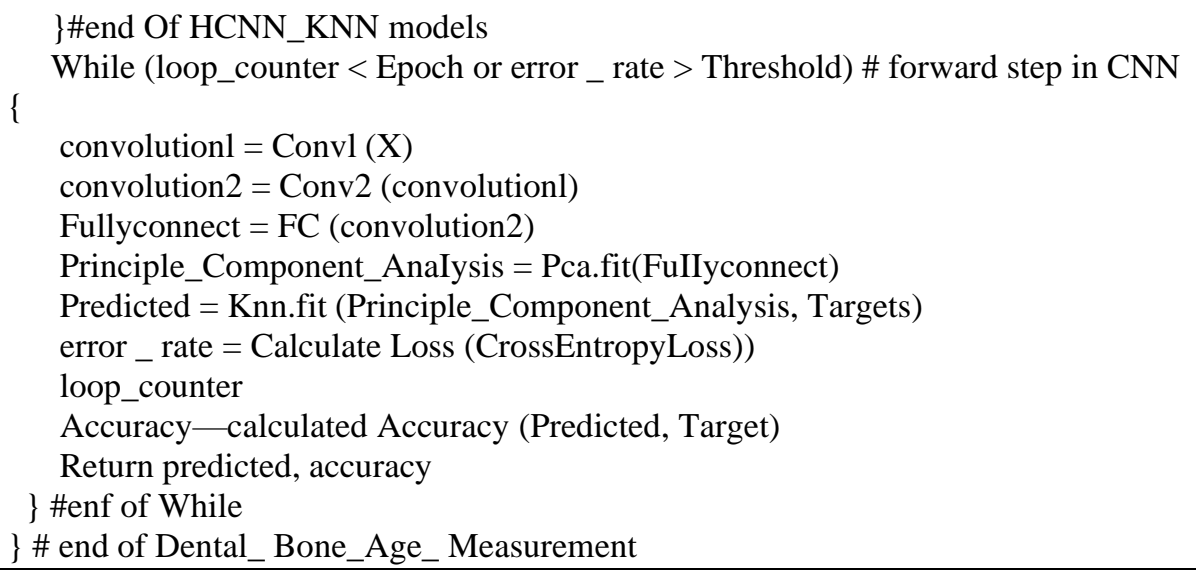

Figure 3. HCNN-KNN Model Algorithm Pseudo-C

\section{Step 4: Evaluating the Proposed Model on Validation Data}

In this step, the optimal amount of weight and bias of the network neurons after network training is transferred to the Convolutional Neural Network in the validation phase. The validation data, segmented according to step 2, is then categorized on the CNN network with optimal parameters. The process of components of the classification algorithm according to the training phase passes through the CNN-PCA-KNN layers. Finally, the identification rate is evaluated with the Accuracy criterion according to the equation in confusion metrics.

Accuracy $=\frac{\mathrm{TP}+\mathrm{TN}}{\mathrm{TP}+\mathrm{FP}+\mathrm{TN}+\mathrm{FN}}$

TP: The number of times the class is positive and correctly detected.

FP: The number of times the class was positive and undiagnosed.

TN: The number of times the class is incorrect (negative) and correctly diagnosed

FN: The number of times the class is incorrect (negative) and not correctly recognized.

\section{Step 5: Validating the Proposed Model on Second Dataset (Test Dataset)}

As mentioned in step 3, the test data is new (not the part of training dataset) and is collected separately from the dataset with different properties. This data combines X-ray OPG images of different races, with different genders and at different ages. This data enters the CNN network as a validation step and is categorized after passing through conv1, pooling1, conv2, pooling2, fully connected layer, PCA layer, and KNN layer. A vital feature of this step is the validation of the proposed model. Achieving acceptable results on various test data will indicate the satisfactory performance of the proposed model. In the following, the Experimental Result section, the data collected on this architecture is evaluated as a robust architecture of CNN networks.

\section{Result and Discussion}

In the following, first, the database used in this article is introduced, then the tools and the framework are introduced. Finally, we evaluated our proposed model on the dataset.

\section{A. Datasets Description}


1 Dental OPG is a panoramic radiograph that scans the upper and lower jaws with a two2 dimensional view that shows a semicircle from ear to ear. The method presented in this article 3 is based on dental OPG data collected from dental teaching institutes and private dental clinics 4 in Malaysia. These collected data were with the different image sizes are resized before 5 importing into the proposed model. The original image size for all data has been changed to $6600 \times 1024$ pixels. Subjects from this study were randomly selected from 1922 patients 7 between 15 and 23 years of age, and this age range determines the age of minors which could 8 help the model find better features and train the model more accurately. On the other hand, we collected the new dataset to use for the validity of the result of the first dataset. The second dataset included 130 random images, and the results of this test data are explained in the experimental Result 4.

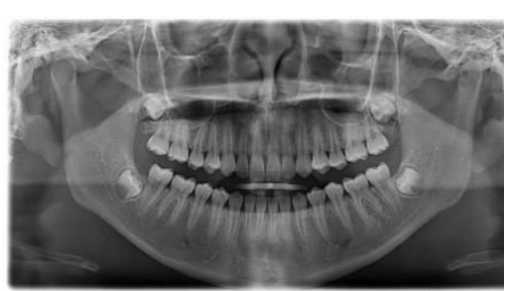

15 years

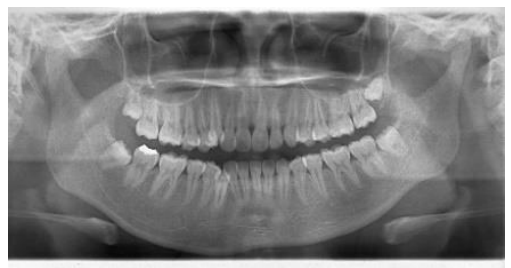

18 years

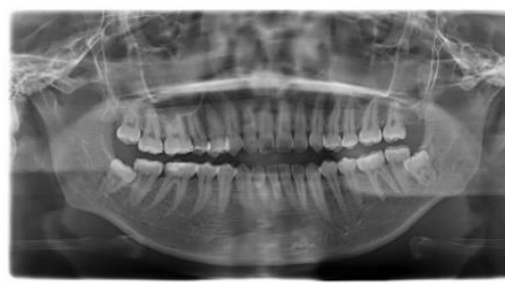

21 years

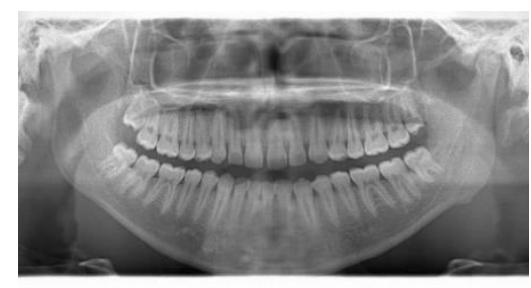

16 years

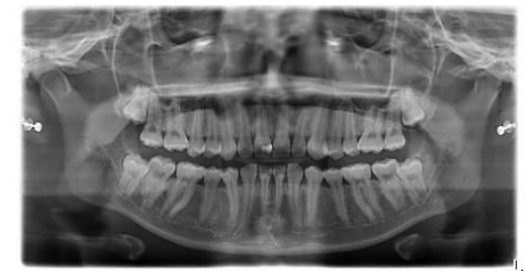

19 years

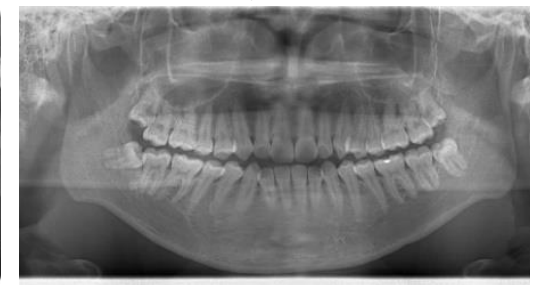

22 years

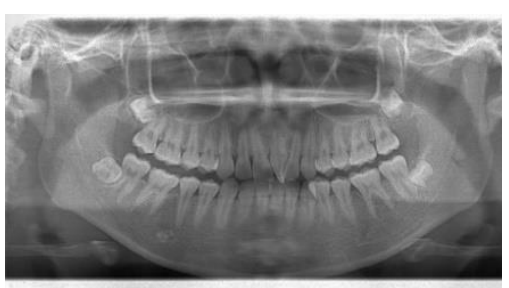

17 years

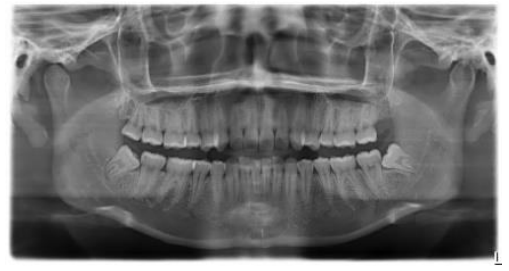

20 years

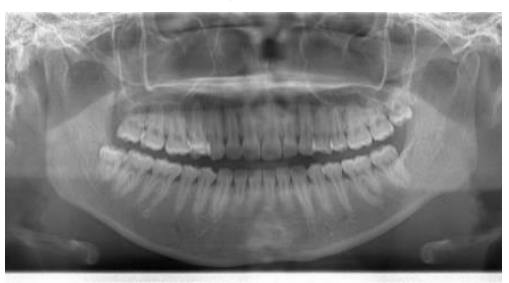

23 years

Figure 4. Representation of Each Age Class.

The collected dataset shows in Figure 4 has a prominent feature as it contains the addition of the categorization of data in the form of age range by year, age range by month, by season (3 months), and six months (Dataset on Year, on \pm six months, on \pm three months, and \pm one month). This classification allows us to evaluate our proposed method on a test database to examine the performance of the proposed method. Figure 5 shows the frequency of data in the database based on each of the Dataset on Year, on \pm six months, on \pm three months, and \pm one month. 


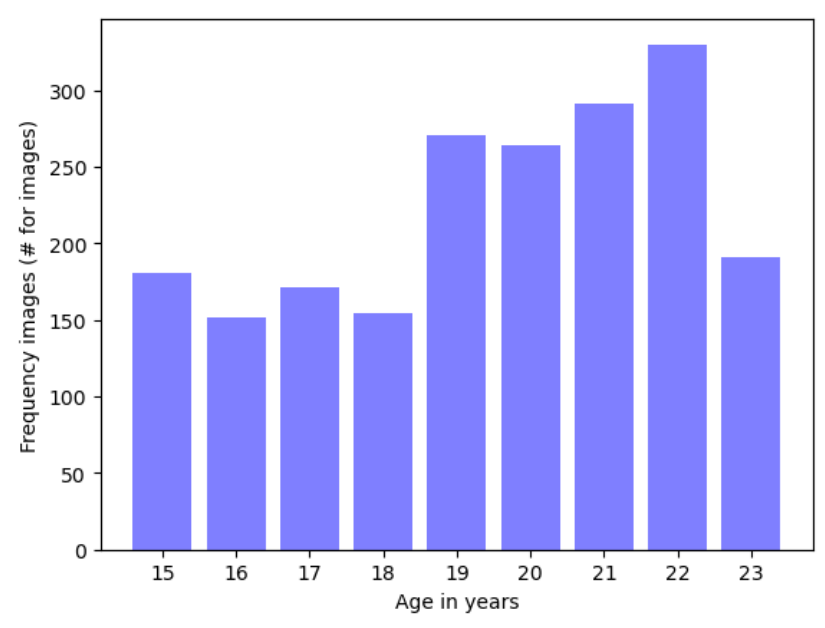

a. Frequency of Dental Images for Years Dataset

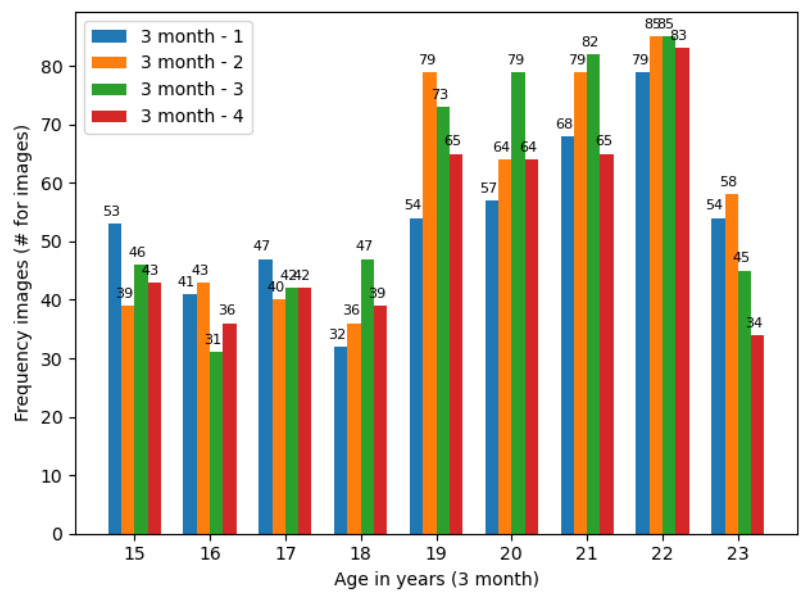

c. Frequency of Dental Images for \pm 3 Months Dataset

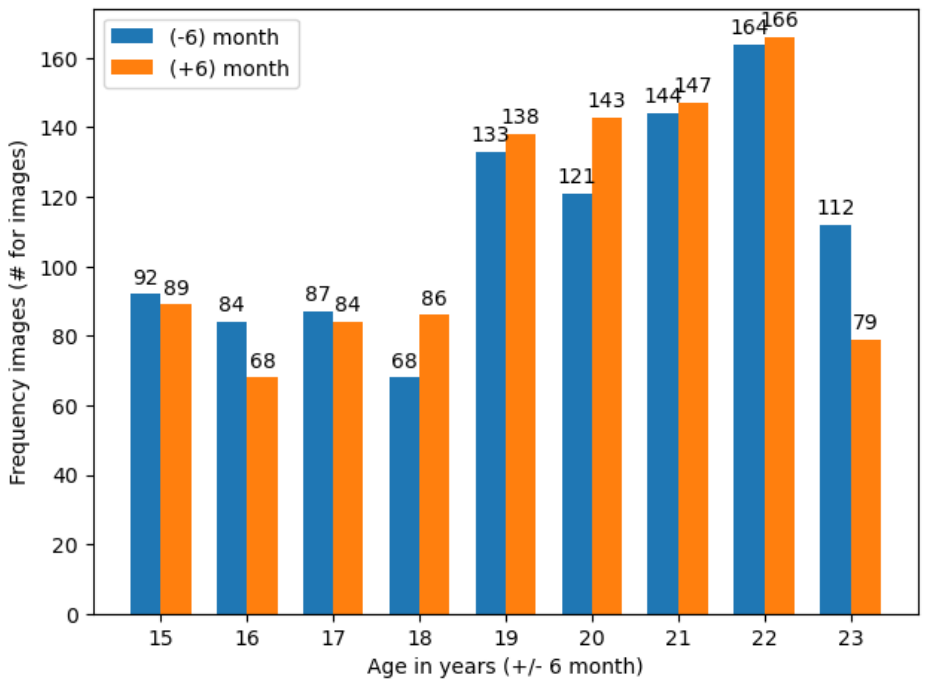

b. Frequency of Dental Images for \pm 6 Months Dataset

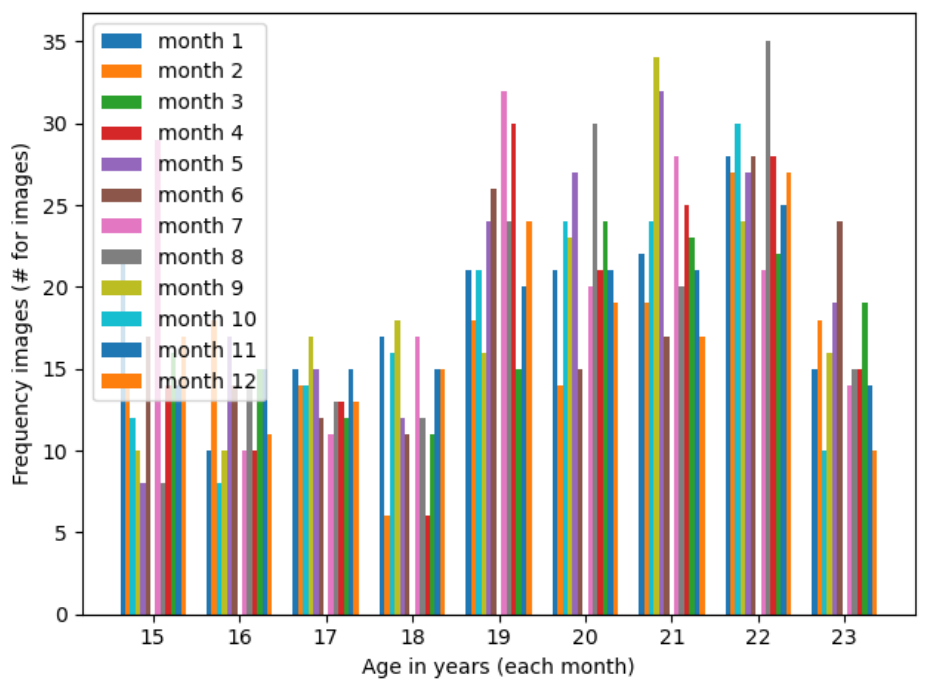

d. Frequency of Dental Images for \pm 1 Months Dataset
8 This study used a machine with NVIDIA GeForce GTX 1080 8GB, 8GB memory, Intel Core 9 i7, and 3.40GHz. We also used libraries NumPy, Matplotlib, Sklearn. Metrics, PyTorch, 10 Torchvision to perform the analyses. 


\section{C. Experiment 1: CNN Model}

2 Table 2 evaluates the results of applying the CNN model to four different states of the dataset.

3 In the first step, the accuracy value for training and valid data is evaluated from the original 4 dataset without any pre-processing and data augmentation. Subsequently, the accuracy value 5 for training and validation dataset from the database is evaluated by pre-processing operations 6 on the original dataset and data augmentation operations.

7 8

Table 2: Experimental Results on a Dental Dataset Based on CNN

\begin{tabular}{|c|c|c|c|c|c|}
\hline \multirow[b]{2}{*}{ Data set } & \multirow[b]{2}{*}{ Train/Valid } & \multicolumn{4}{|c|}{ Accuracy $\%$} \\
\hline & & $\begin{array}{l}\text { Dataset on } \\
\text { Year }\end{array}$ & $\begin{array}{l}\text { Dataset on } \pm 6 \text { mo } \\
\text { nth }\end{array}$ & $\begin{array}{l}\text { Dataset on } \pm 3 \text { mo } \\
\text { nth }\end{array}$ & $\begin{array}{l}\text { Dataset on } \pm 1 \mathrm{mo} \\
\text { nth }\end{array}$ \\
\hline \multirow[t]{2}{*}{$\overline{\text { Original }}$} & Train Accuracy & 77.85 & 76.24 & 72.41 & 70.26 \\
\hline & Valid Accuracy & 58.91 & 43.72 & 38.75 & 23.29 \\
\hline \multirow{2}{*}{$\begin{array}{l}\text { Augmented- } \\
\text { preprocessed }\end{array}$} & Train Accuracy & 98.84 & 98.58 & 98.75 & 96.99 \\
\hline & Valid Accuracy & 97.43 & 98.44 & 98.13 & 95.62 \\
\hline
\end{tabular}

9

10 The consistency value on data conducted with pre-processing and data augmentation operations

11 has given more suitable performance as shown in Table 1. Figure 6 displays the loss reduction 12 mechanism for four separate problem modes (Dataset on Year, \pm 6 months, \pm 3 months, and \pm 1 13 months) with 4000 repetitions (epoch equivalent to 10 with about 400 batch size 16). It should 14 be noted that the results obtained on cross-validation with 20-80 segmentation are on dataset 15 images. As a result, $20 \%$ of the database images are devoted to validation, while the remaining $1680 \%$ are dedicated to training. Therefore, for a more accurate evaluation, the proposed cross17 validation models with divisions of 30-70 and 50-50 have been evaluated in the 'Experimental 18 result 4' section.

19
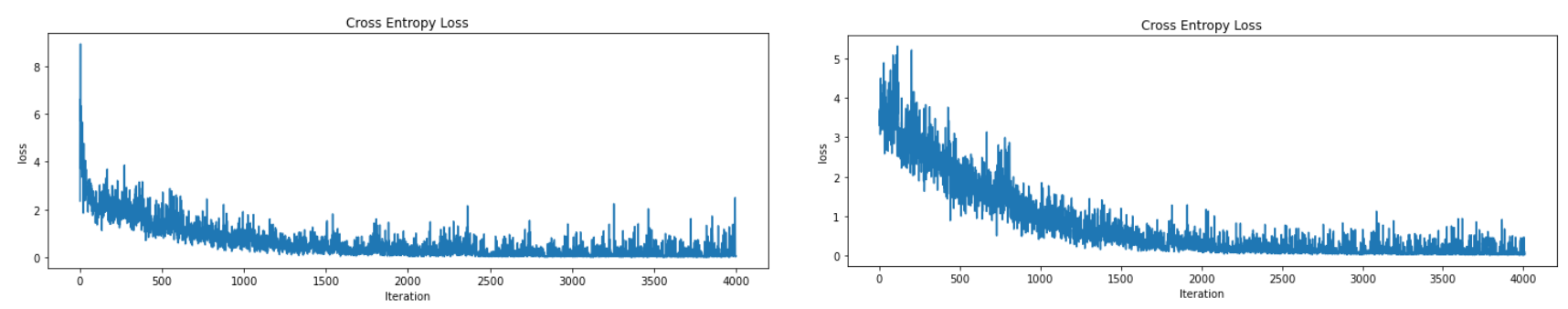

a. Error Loss for Dataset Years

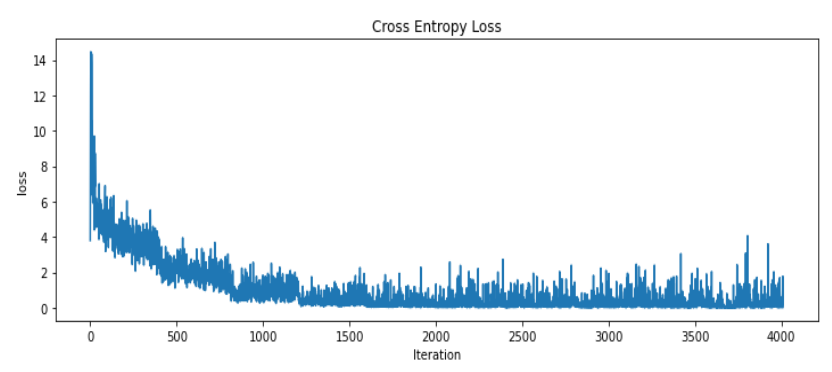

b. Error Loss for a Dataset with \pm 6 Months

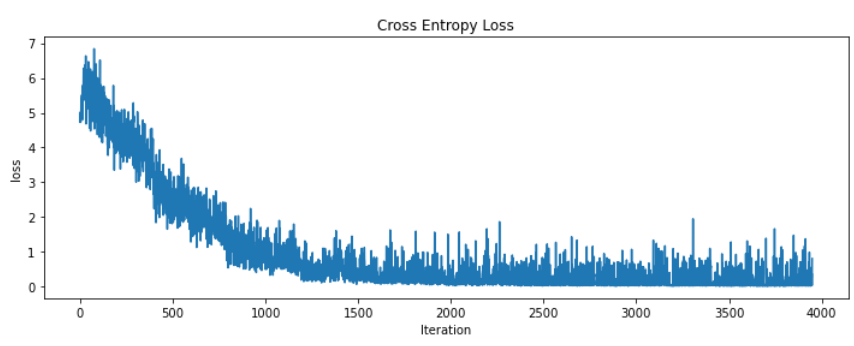

c. Error Loss for a Dataset with \pm 3 Months

d. Error Loss for a Dataset with \pm 1 Months 

6

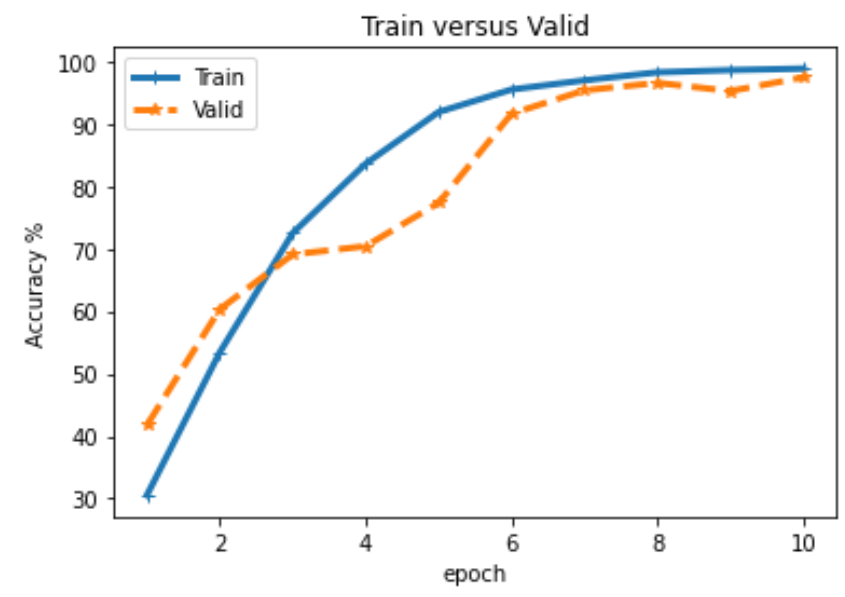

a. Test Versus Valid for the Dataset Years

7

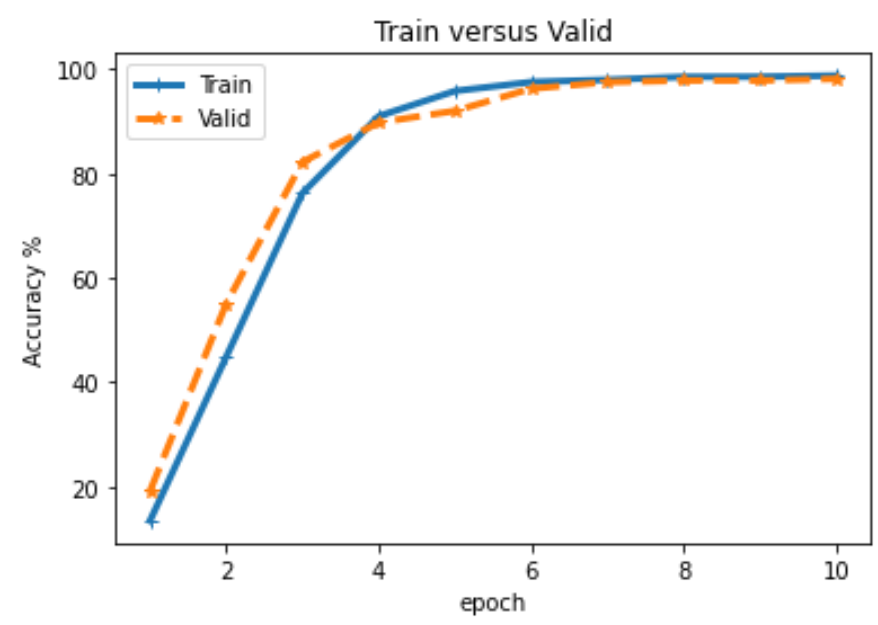

8

c. Test Versus Valid for the Dataset with \pm 3 Months

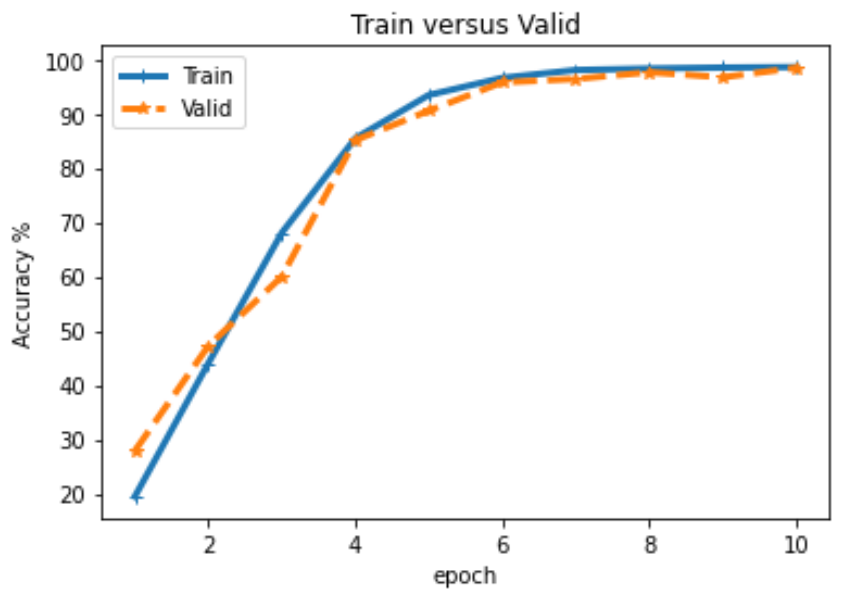

b. Test Versus Valid for the Dataset with \pm 6 Months

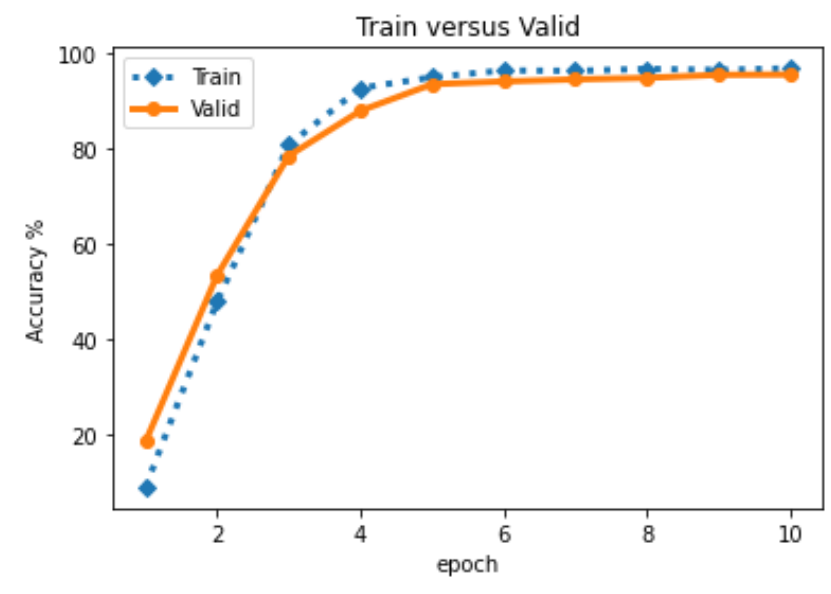

d. Test Versus Valid for the Dataset

Figure 7. Evaluation of Accuracy (Train Versus Valid) in Different Epoch Based on the CNN Model on Four States of the Dataset (a. Dataset on Year, b. on \pm 6 -Months, c. on \pm 3 Months, and d. on \pm 1 Month).

Therefore, the upward trend inaccuracy at ten epochs indicates an increase in CNN learning. Figure 7a) shows accuracy on data based on year, b) show accuracy on data based on \pm 6 months, c) show accuracy on data based on \pm three months and d) display accuracy on data based on one month.

\section{Experiment 2: HCNN-KNN Model}

As mentioned in the proposed model section, the combined methodology of convolutional neural networks and the nearest neighbour (classification) is used in this study. In this proposed 
1 hybrid model (HCNN-KNN), the input data passes through the three main layers of CNN,

2 PCA, and KNN methods, respectively, and is finally categorized by KNN.

3 The results show that the KNN classification with 500 features selected from 'FullyPCA' (PCA 4 data transform after fully connected in $\mathrm{CNN}$ ) has an acceptable outcome. As shown in Table 53 , the accuracy on the dataset on Year, dataset on \pm 6 -months, dataset on \pm 3 -months, and 6 dataset on \pm 1 -month are equivalent to $99.98,99.96,99.87$, and 98.78 , respectively and, the 7 accuracy for train data is equal to 100. This means that the network is fully trained on train 8 data. It is noteworthy that the results obtained for $\mathrm{k}=1$ in the proposed HCNN-KNN hybrid 9 algorithm.

10 In this experiment, the HCNN-KNN model was evaluated for bone age measurement.

Table 3: Experimental Results on Dental Dataset Based on HCNN-KNN

\begin{tabular}{llcccc} 
& & \multicolumn{5}{c}{ Accuracy $\%$} \\
\cline { 3 - 6 } Data set & Train/Valid & Dataset on Year & $\begin{array}{l}\text { Dataset on } \pm 6 \text { mont } \\
\mathrm{h}\end{array}$ & $\begin{array}{l}\text { Dataset on } \pm 3 \text { mont } \\
\mathrm{h}\end{array}$ & $\begin{array}{l}\text { Dataset on } \pm 1 \text { mont } \\
\mathrm{h}\end{array}$ \\
\hline \hline Original & Train Accuracy & 100 & 100 & 100 & 100 \\
& Valid Accuracy & 99.93 & 99.84 & 99.74 & 98.15 \\
\multirow{2}{*}{ Augmented } & Train Accuracy & 100 & 100 & 100 & 100 \\
& Valid Accuracy & 99.98 & 99.96 & 99.87 & 98.78 \\
\hline
\end{tabular}

13

14 Figure 8 shows the accuracy and error loss in the combined HCNN-KNN model for $\mathrm{k}=1$ and 1510 epochs on the train and valid data.

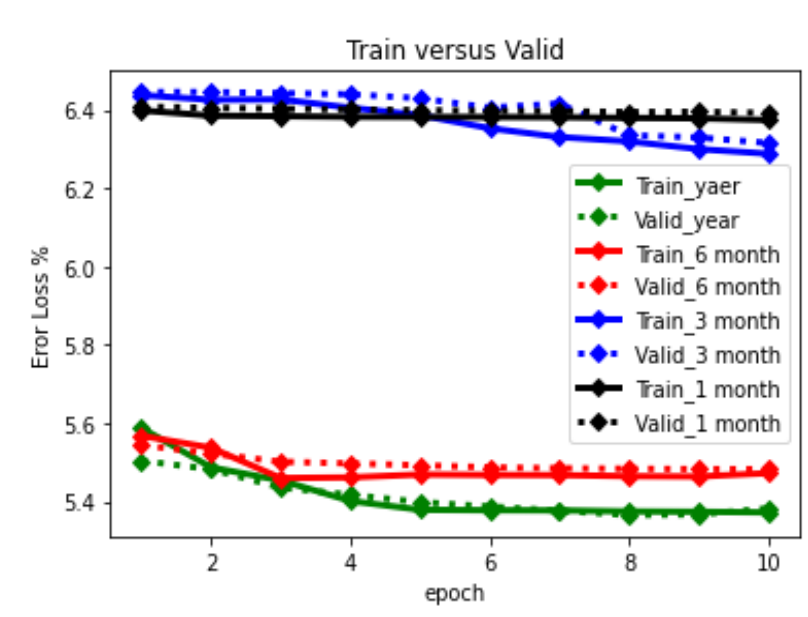

a. Error loss for HCNN-KNN

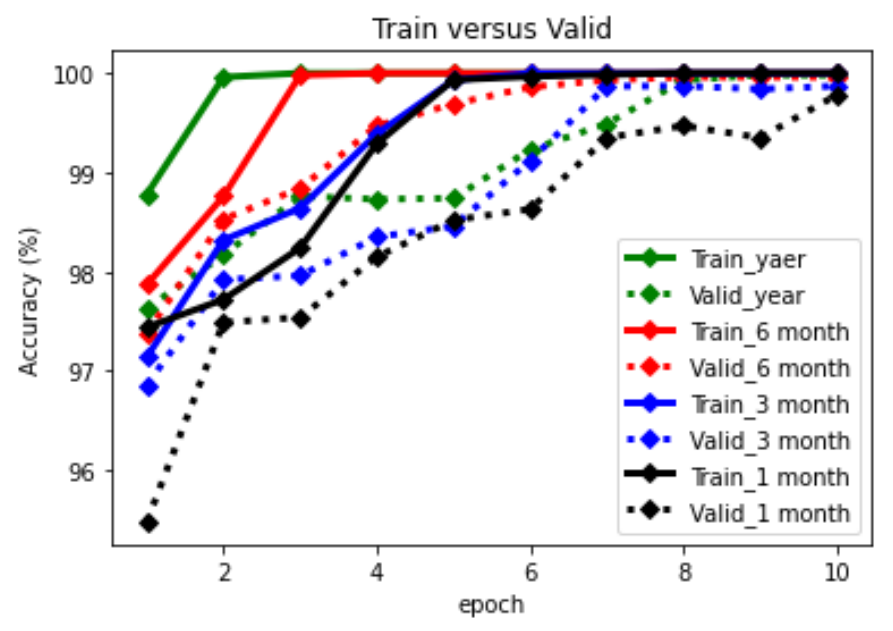

b. Accuracy for HCNN-KNN 
1 To evaluate the KNN method in the proposed HCNN-KNN hybrid model, k equivalent 2 and 23 have been used in evaluating the proposed model. Table 4 shows the accuracy results of $\mathrm{k}=$ $31,2,3$ on the validation data.

4

5

\begin{tabular}{lcccc}
\hline & \multicolumn{4}{c}{ Accuracy $\%$} \\
\cline { 2 - 5 } $\mathrm{K}$ & Dataset on \pm 1 year & Dataset on \pm 6 month & Dataset on \pm 3 month & Dataset on \pm 1 month \\
\hline \hline $\mathrm{K}=1$ & 99.98 & 99.96 & 99.87 & 98.78 \\
$\mathrm{~K}=2$ & 59.26 & 55.61 & 52.64 & 51.20 \\
$\mathrm{~K}=3$ & 43.89 & 41.64 & 38.02 & 33.77 \\
\hline
\end{tabular}

6

7 In analysing the value of $\mathrm{k}$ in the nearest neighbourhood and avoiding overfitting for small $\mathrm{ks}$ 8 such as $\mathrm{k}=1$ and underfitting for large $\mathrm{k}$, use new dental images separate from the data in the 9 dataset and have special features. The special features of these images are related to different 10 races, different genders and are selected in the age range of 15 to 23. Obtaining acceptable 11 results on these data indicates the proper performance of the KNN classifier for $\mathrm{k}=1$ and from 12 overfitting in the bone age measurement problem.

\section{E. Experiment 3: Evaluation of Different Cross-Validation}

Table 5: Evaluation of Accuracy on Different Cross-Validation on a Dataset

\begin{tabular}{|c|c|c|c|c|c|}
\hline \multirow[b]{2}{*}{ Dataset Type } & \multirow[b]{2}{*}{ Methods } & \multirow[b]{2}{*}{ Train/Valid } & \multicolumn{3}{|c|}{ Accuracy on different Cross-Validation (valid-Train) } \\
\hline & & & 20-80 & $30-70$ & $50-50$ \\
\hline \multirow{6}{*}{$\begin{array}{l}\text { Dataset on } \\
+1 \text { Year }\end{array}$} & $\mathrm{CNN}$ & Train & 98.84 & 98.16 & 97.20 \\
\hline & & Valid & 97.43 & 94.08 & 85.58 \\
\hline & CNN-KNN & Train & 100.00 & 100.00 & 100 \\
\hline & & Valid & 99.98 & 99.97 & 99.95 \\
\hline & Resnet (Deep model) & Train & 99.04 & 98.83 & 98.80 \\
\hline & & Valid & 98.25 & 95.75 & 86.03 \\
\hline \multirow{6}{*}{$\begin{array}{c}\text { Dataset on } \\
\pm 6 \text { month }\end{array}$} & $\mathrm{CNN}$ & Train & 98.58 & 98.50 & 96.75 \\
\hline & & Valid & 98.44 & 94.72 & 86.66 \\
\hline & CNN-KNN & Train & 100.00 & 100.00 & 100 \\
\hline & & Valid & 99.96 & 99.78 & 99.78 \\
\hline & Resnet (Deep model) & Train & 98.66 & 98.53 & 98.42 \\
\hline & & Valid & 98.69 & 95.47 & 87.43 \\
\hline \multirow{4}{*}{$\begin{array}{l}\text { Dataset on } \\
\pm 3 \text { month }\end{array}$} & $\mathrm{CNN}$ & Train & 98.75 & 98.48 & 97.77 \\
\hline & & Valid & 98.13 & 96.25 & 84.61 \\
\hline & CNN-KNN & Train & 100.00 & 100.00 & 100.00 \\
\hline & & Valid & 99.87 & 99.82 & 99.68 \\
\hline
\end{tabular}




\begin{tabular}{|c|c|c|c|c|c|}
\hline & Resnet (Deep model) & $\begin{array}{l}\text { Train } \\
\text { Valid }\end{array}$ & $\begin{array}{l}98.69 \\
98.44\end{array}$ & $\begin{array}{l}98.74 \\
96.42\end{array}$ & $\begin{array}{l}98.70 \\
86.23\end{array}$ \\
\hline \multirow{6}{*}{$\begin{array}{l}\text { Dataset on } \\
\pm 1 \text { month }\end{array}$} & $\mathrm{CNN}$ & Train & 96.99 & 97.12 & 94.46 \\
\hline & & Valid & 95.62 & 92.40 & 80.32 \\
\hline & CNN-KNN & Train & 100.00 & 100.00 & 100.00 \\
\hline & & Valid & 98.78 & 98.52 & 98.29 \\
\hline & Resnet (Deep model) & Train & 97.25 & 97.24 & 97.15 \\
\hline & & Valid & 95.63 & 93.16 & 90.27 \\
\hline
\end{tabular}

1

2

3

4

5

6

7

8

\section{F. Experiment 4: Evaluation of Proposed Model on Second Dataset (Test Dataset)}

The key feature of this experiment is the validation of the proposed model. A set of new images with different statistical populations has been used to validate the proposed model. These images are not available in the first dataset and are a combination of X-Ray OPG images with different races (Malay, Indian, Chinese), with distinct genders and at various range of ages and with other conditions than ordinary dentistry (orthodontic and malignant images). This statistical population includes 130 new dental X-Ray OPG collected to solve the dental age accuracy problem. Figure 9 shows a sample of data outside the first dataset and effectively improves the age estimation accuracy even in malignant dental images of the proposed model.
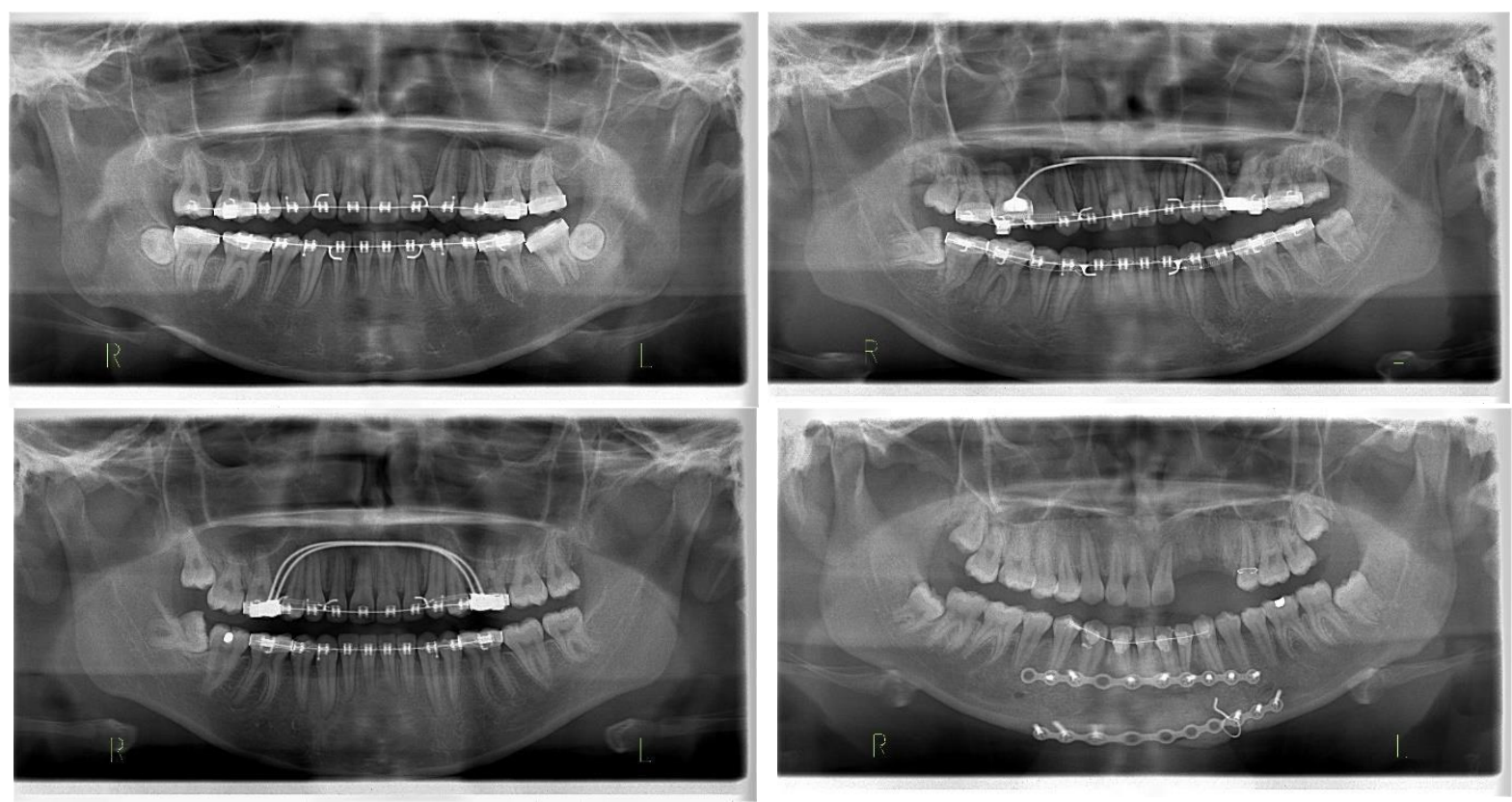

Figure 9. Different Images of Test Data to Evaluate the Orthodontics (Malignant) of the Proposed Model.

As Table 6 shows, the proposed HCNN-KNN model has obtained better results on the test data. Obtaining satisfactory results on a new dataset outside the dataset images indicates the performance of the proposed model. 
Table 6: Accuracy Result of The Proposed HCNN-KNN Model on Test New Dataset

\begin{tabular}{llrrr} 
& & CNN & \multicolumn{2}{c}{ CNN-KNN } \\
\cline { 3 - 5 } & Test data (130 dental images) & 22.93 & K=1 & $\mathbf{9 9 . 2 3}$ \\
& & & $\mathrm{K}=2$ & 74.61 \\
& & & $\mathrm{~K}=3$ & 70.22 \\
\hline Bll test data & Malay race & 25.23 & & $\mathrm{~K}=1$ \\
& (71 images) & & & 100 \\
& & & $\mathrm{~K}=2$ \\
& & & 76.05 \\
& & & & $\mathrm{~K}=3$ \\
& Indian race & & & 73.23 \\
\cline { 3 - 5 } & (16 images) & 20.61 & $\mathrm{~K}=1$ & 93.75 \\
& & & $\mathrm{~K}=2$ & 75.00 \\
& Chinese race & & $\mathrm{K}=3$ & 68.75 \\
\cline { 3 - 5 } & (43 images) & 21.37 & $\mathrm{~K}=1$ & 97.67 \\
& & $\mathrm{~K}=2$ & 72.09 \\
& & & $\mathrm{~K}=3$ & 69.76 \\
\hline
\end{tabular}

2

3 According to Table 6, The results of this experiment have been evaluated on a test dataset with 4 the form of different races. HCNN-KNN effects have also been tested for $\mathrm{k} 1,2$, and 3 . The 5 results show the proper performance of the proposed model for $\mathrm{k}=1$.

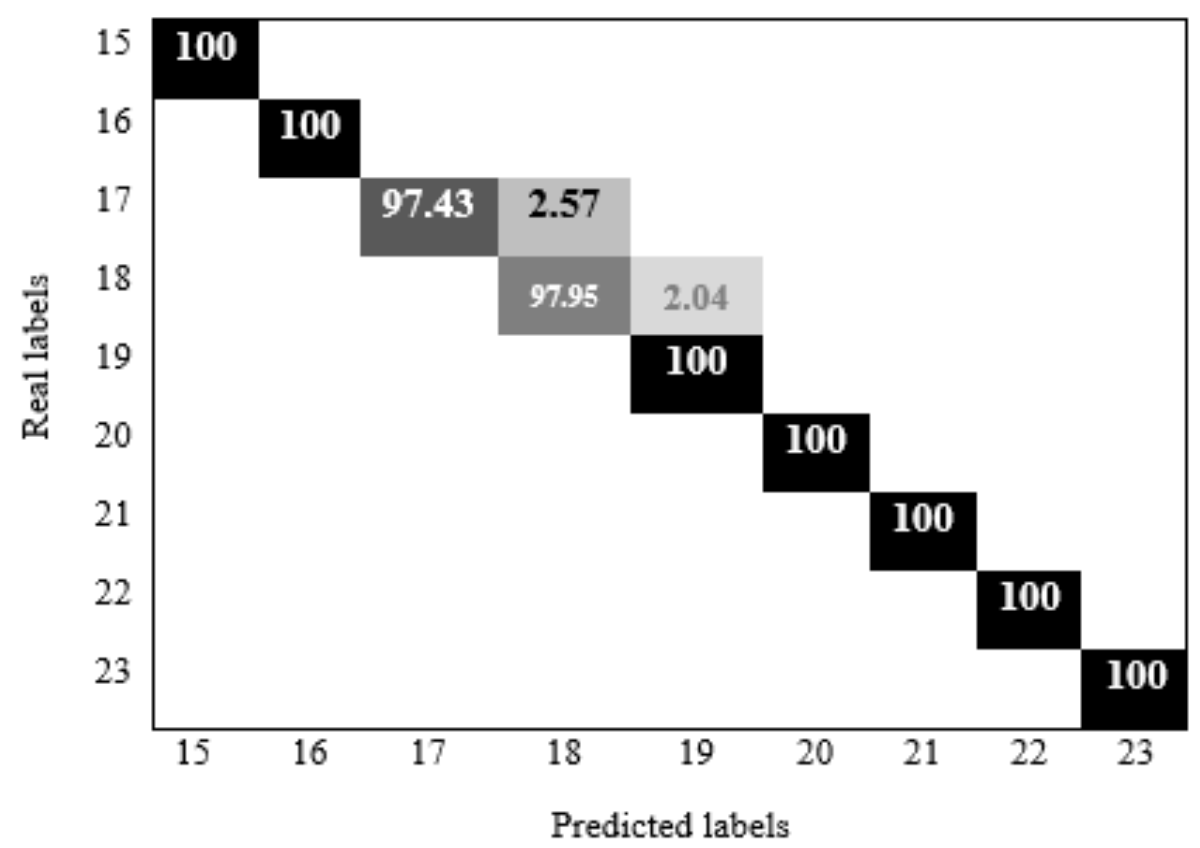

Figure 10. A Confusion Matrix of Hybrid HCNN-KNN Proposed Model Application on Enhancement Dataset.

Figure 10 shows the confusion matrix related to the test dataset classification based on the HCNN- KNN model. For example, as seen in the confusion matrix, 97.95 percent of the 18 -year-old age range was correctly diagnosed, and 2.04 percent was incorrectly identified as the 19-year-old. 


\section{G. Experimental Result 5: Compared with Other Methods}

Finally, an attempt was made in the last experiment to compare the results obtained in this study with the currently existing models.

Table 7: Comparison Between the Proposed HCNN-KNN Model and Other Studies in BAM based on Dental Images.

\begin{tabular}{|c|c|c|}
\hline Models & $\begin{array}{c}\text { Accuracy } \\
\%\end{array}$ & References \\
& $\mathbf{9 7 . 4 3}$ & Our Other Test \\
\hline CNN & $\mathbf{9 9 . 9 8}$ & Proposed Hybrid Model \\
\hline CNN-KNN & $\mathbf{9 8 . 2 5}$ & Our Other Test \\
\hline ResNet & $\mathbf{5 5 . 6 9}$ & Our Other Test \\
\hline GoogLeNet Inception & 92.1 & (Houssein, 2020) \\
\hline SVM & 89.00 & (Hemalatha, 2020) \\
\hline FNN-TLBO & 90.00 & Avuçlu, 2020) \\
\hline Multilayer Perceptron & &
\end{tabular}

The collected data along with the proposed model have been tested with other models such as ResNet, CNN, GoogLeNet Inception. As Table 7 shows, the hybrid HCNN-KNN model (HCNN-KNN) has obtained much better accuracy compared to other classification algorithms.

No reported research has been done on \pm six months, on \pm three months, and \pm one month so far, but only compared by the year. However, the results of this proposed model are compared with previous studies by years of accuracy. For example, Avuçlu used the Multilayer Perceptron model with 1315 numbers of dental OPG images, and the highest accuracy was $90 \%$ just for accuracy of the years. Also, in 2020, Hussein used the SVM algorithm for a population of 1429 dental radiographs, and they only achieved 92\% accuracy for years. Therefore, based on Table 7, the results of the comparative studies for accuracy by years are still below the accuracy achieved in this study which is 99.98 .

\section{Conclusions}

This novel investigation implemented for the first time with precision to the range of $+/-6$ months developed an HCNN-KNN model, which uses for BAM to increase the existing model's accuracy and prevent $\mathrm{CNN}$ overload situations. We considered more specific cases of bone age measurement with the help of Dental X-Ray OPG images to solve the problem of bone age measurement in determining the age range of 15 to 23 years based on the Year, \pm six months, \pm three months, and \pm one month. We used an integrative methodology of convolutional neural networks (as extraction and analysis of information and features in dental images) and the nearest neighbour (classification method). The main purpose of this proposed model is to use KNN instead of SoftMax in the fully connected layer to increase the performance of convolutional networks in the classification phase. Using principal component analysis as data transform and feature dimensionality reduction in a fully connected layer and before classifying KNN as Fully_PCA. The main purpose of this method is to transfer data to 
1 space with more specific information to increase the performance of the classification phase.

2 The model achieved the accuracy of $99.98,99.96,99.87$, and 98.78 on one year, \pm 6 -month, \pm 3 3-month, and \pm 1 -month range respectively. Our proposed method evaluated different cross4 validation of the dataset. 50-50 train-valid, 70-30 train-valid and 20-80 train-valid cross5 validation on dataset. Evaluating the proposed model on a new dataset with different races also 6 proved superior performance of the model. The benchmarking with current existing models 7 also showed that the HCNN-KNN model is the best model for bone age measurement.

\section{$9 \quad$ Author Contribution}

FS and SSB designed the study; FS wrote the manuscript. FS, SSB, AB, and HAR edited the manuscript. FS collected the data. FS carried out the analyses, including statistical analyses and the implementation of machine learning methods. FS generated all figures and tables. HAR was not involved in any analysis. All authors have read and approved the final version of the manuscript.

\section{Conflict of interests}

The authors declare no competing financial and non-financial interests.

\section{Acknowledgment}

We are thankful to the school of computer science and engineering, SCE Taylor's University, for providing the scholarship to complete the studies. HAR is supported by the UNSW Scientia Program Fellowship and the Australian Research Council Discovery Early Career Researcher Award (DECRA).

\section{References}

1. Albawi. (2017). Understanding of a convolutional neural network. Paper presented at the 2017 International Conference on Engineering and Technology (ICET).

2. Albawi, S. M., (2017). Understanding of a convolutional neural network. Paper presented at the 2017 International Conference on Engineering and Technology (ICET).

3. Alkaabi, S., Yussof, Salman, Al-Mulla, Sameera. (2019). Evaluation of Convolutional Neural Network based on Dental Images for Age Estimation. Paper presented at the 2019 International Conference on Electrical and Computing Technologies and Applications (ICECTA).

4. Amon, J. J. (2020). COVID-19 and detention. Health and Human Rights, 22(1), 367-370. 
5. Asif, M. K. (2019). Dental age estimation in Malaysian adults based on volumetric analysis of pulp/tooth ratio using CBCT data. Legal Medicine, 36, 50-58.

6. Avuçlu. (2020). The determination of age and gender by implementing new image processing methods and measurements to dental X-ray images. Measurement, 149, 106985.

7. Avuçlu, E., Başçiftçi, Fatih (2020). The determination of age and gender by implementing new image processing methods and measurements to dental X-ray images. Measurement, 149,106985 .

8. Bagattoni, S. (2019). Applicability of Demirjian's method for age estimation in a sample of Italian children with Down syndrome: A case-control retrospective study. Forensic science international, 298, 336-340.

9. Banar, N. (2020). Towards fully automated third molar development staging in panoramic radiographs. International journal of legal medicine, 134(5), 1831-1841Blatt, S., Michael, Amy. (2020). Forensic Imaging.

10. Cameriere, R. (2019). Carpals and epiphyses of radius and ulna as age indicators using longitudinal data: a Bayesian approach. International journal of legal medicine, 133(1), 197204.

11. Cascianelli, S. B.-C. (2018). Dimensionality reduction strategies for CNN-based classification of histopathological images. Paper presented at the international conference on intelligent interactive multimedia systems and services.

12. Clark, A., Jit, M., Warren-Gash, C., Guthrie, B., Wang, H. H., Mercer, S. W., Ong, K. L. (2020). Global, regional, and national estimates of the population at increased risk of severe COVID-19 due to underlying health conditions in 2020: a modelling study. The Lancet Global Health, 8(8), e1003-e1017.

13. Čular, L. (2017). Dental age estimation from panoramic X-ray images using statistical models. Paper presented at the Proceedings of the 10th International Symposium on Image and Signal Processing and Analysis.

14. Dahlberg. (2019). A systematic review of the agreement between chronological age and skeletal age based on the Greulich and Pyle atlas. European radiology, 29(6), 2936-2948.

15. Parvin, H., Parvin, S. (2011). Divide and conquer classification. Australian Journal of Basic and Applied Sciences, 5(12), 2446-2452.

16. De Tobel, J. (2017). An automated technique to stage lower third molar development on panoramic radiographs for age estimation: a pilot study. The Journal of forensic odontostomatology, 35(2), 42.

17. De Tobel, J. (2020). Multi-factorial age estimation: A Bayesian approach combining dental and skeletal magnetic resonance imaging. Forensic science international, 306, 110054.

18. Ezhil, I. (2018). Estimation of age from physiological changes of teeth. Drug Invention Today, 10.

19. Farhadian, M., Salemi, Fatemeh, Saati, Samira, Nafisi, Nika. (2019). Dental age estimation using the pulp-to-tooth ratio in canines by neural networks. Imaging science in dentistry, 49(1), 19. 
20. Ginnis, J. (2019). Measurement of early childhood oral health for research purposes: dental caries experience and developmental defects of the enamel in the primary dentition Odontogenesis (pp. 511-523): Springer.

21. Javanmard, R., JeddiSaravi, K., (2013). Proposed a new method for rules extraction using artificial neural network and artificial immune system in cancer diagnosis. Journal of Bionanoscience, 7(6), 665-672.

22. Glorot, X. B., Yoshua. (2010). Understanding the difficulty of training deep feedforward neural networks. Paper presented at the Proceedings of the thirteenth international conference on artificial intelligence and statistics.

23. Gurpinar, F. (2016). Kernel ELM and CNN based facial age estimation. Paper presented at the Proceedings of the IEEE conference on computer vision and pattern recognition workshops.

24. Haque, I. R. I., Neubert, Jeremiah. (2020). Deep learning approaches to biomedical image segmentation. Informatics in Medicine Unlocked, 18, 100297.

25. Hemalatha. (2020). A versatile approach for dental age estimation using fuzzy neural network with teaching learning-based optimization classification. Multimedia Tools and Applications, 79(5), 3645-3665.

26. Hemalatha, B., Rajkumar, N. (2021). A modified machine learning classification for dental age assessment with effectual ACM-JO based segmentation. International Journal of BioInspired Computation, 17(2), 95-104.

27. Ahmadinia, M., Meybodi, M. R., Esnaashari, M., (2013). Energy efficient and multi-stage clustering algorithm in wireless sensor networks using cellular learning automata. IETE Journal of Research, 59(6), 774-782.

28. Hinton, G. E. S., (2012). Improving neural networks by preventing co-adaptation of feature detectors. arXiv preprint arXiv:1207.0580.

29. Houssein. (2020). Dental age estimation based on X-ray images. Computers, Materials \& Continua, 62(2), 591-605.

30. Houssein, E. H. (2020). Dental age estimation based on X-ray images. Computers, Materials \& Continua, 62(2), 591-605.

31. Jarrett, K., Kavukcuoglu, Koray, Ranzato, Marc'Aurelio, LeCun, Yann. (2009). What is the best multi-stage architecture for object recognition? Paper presented at the 2009 IEEE 12th international conference on computer vision.

32. Kahaki, S. M. (2020). Deep convolutional neural network designed for age assessment based on orthopantomography data. Neural Computing and Applications, 32(13), 9357-9368. Kalantari. (2018). Computational intelligence approaches for classification of medical data: State-of-the-art, future challenges and research directions. Neurocomputing, 276, 2-22.

33. Kapadia, F., Stevens, J., \& Silver, D. (2020). Dental Radiographs for Age Estimation in US Asylum Seekers: Methodological, Ethical, and Health Issues. American Journal of Public Health, 110(12), 1786-1789. 
34. Alinejad-Rokny, H., Anwar, F., Waters, S. A., Davenport, M. P., \& Ebrahimi, D. (2016). Source of CpG depletion in the HIV-1 genome. Molecular biology and evolution, 33(12), 3205-3212.

35. Kim, J. (2019). DeNTNet: Deep Neural Transfer Network for the detection of periodontal bone loss using panoramic dental radiographs. Scientific reports, 9(1), 1-9.

36. Kramer, O. (2013). K-nearest neighbors Dimensionality reduction with unsupervised nearest neighbors (pp. 13-23): Springer.

37. Krizhevsky. (2012). Imagenet classification with deep convolutional neural networks. Advances in neural information processing systems, 25, 1097-1105.

38. Krizhevsky, A., Sutskever, Ilya, Hinton, Geoffrey E. (2017). ImageNet classification with deep convolutional neural networks. Communications of the ACM, 60(6), 84-90.

39. Kruessmann, T. (2021). Internal Investigations in Compliance Matters: What Role for Legal Professional Privilege in Europe? European Business Law Review, 32(2).

40. LeCun, Y. (1989). Backpropagation applied to handwritten zip code recognition. Neural computation, 1(4), 541-551.

41. LeCun, Y. (1998). Gradient-based learning applied to document recognition. Proceedings of the IEEE, 86(11), 2278-2324.

42. Lee, H., Tajmir, S., Lee, J., Zissen, M., Yeshiwas, B. A., Alkasab, T. K., Do, S. (2017). Fully automated deep learning system for bone age assessment. Journal of digital imaging, 30(4), 427-441.

43. Li, J. (2020). Suphx: Mastering mahjong with deep reinforcement learning. arXiv preprint arXiv:2003.13590.

44. Mahmoudi, M. R., Akbarzadeh, H., Parvin, H., Nejatian, S., Rezaie, V., \& Alinejad-Rokny, H. (2021). Consensus function based on cluster-wise two level clustering. Artificial Intelligence Review, 54(1), 639-665.

45. Maas, A. L. H., Awni Y Ng, Andrew Y. (2013). Rectifier nonlinearities improve neural network acoustic models. Paper presented at the Proc. icml.

46. McGill, D. (2021). Bone Age Endocrine Conditions in Pediatrics (pp. 215-218): Springer.

47. Mutasa. (2018). MABAL: a novel deep-learning architecture for machine-assisted bone age labeling. Journal of digital imaging, 31(4), 513-519.

48. Pereira, S. (2016). Brain tumor segmentation using convolutional neural networks in MRI images. IEEE transactions on medical imaging, 35(5), 1240-1251.

49. Qummar, S. (2019). A deep learning ensemble approach for diabetic retinopathy detection. IEEE Access, 7, 150530-150539.

50. Satterstrom, F. K., Kosmicki, J. A., Wang, J., Breen, M. S., De Rubeis, S., An, J.Y., Klei, L. (2020). Large-scale exome sequencing study implicates both developmental and functional changes in the neurobiology of autism. Cell, 180(3), 568-584. e523.

51. Shamshirband. (2019). A survey of deep learning techniques: application in wind and solar energy resources. IEEE Access, 7, 164650-164666. 
52. Shamshirband, S., Fathi, Mahdis, Dehzangi, Abdollah, Chronopoulos, Anthony Theodore, Alinejad-Rokny, Hamid. (2020). A Review on Deep Learning Approaches in Healthcare Systems: Taxonomies, Challenges, and Open Issues. Journal of Biomedical Informatics, 113, 103627.

53. Sharifonnasabi, F., Jhanjhi, N. Z., John, J., Alaboudi, A., \& Nambiar, P. (2020) A Review on Automated Bone Age Measurement Based on Dental OPG Images.

54. Shen, X. (2021). Research on Bone Age Automatic Judgment Algorithm Based on Deep Learning and Hand X-ray Image. Journal of Medical Imaging and Health Informatics, 11(1), 156-161.

55. Hosseinpoor, M., Parvin, H., Nejatian, S., Rezaie, V., Bagherifard, K., Dehzangi, A. (2020). Proposing a novel community detection approach to identify cointeracting genomic regions. Mathematical Biosciences and Engineering, 17(3), 2193-2217.

56. Shi, F., Wang, J., Shi, J., Wu, Z., Wang, Q., Tang, Z., Shen, D. (2020). Review of artificial intelligence techniques in imaging data acquisition, segmentation, and diagnosis for Covid19. IEEE reviews in biomedical engineering.

57. Shobha Rani. (2021). Chronological age assessment based on wrist radiograph processingSome novel approaches. Journal of Intelligent \& Fuzzy Systems (Preprint), 1-13.

58. Sironi, E., Taroni, F., Baldinotti, C., Nardi, C., Norelli, G.-A., Gallidabino, M., \& Pinchi, V. (2018). Age estimation by assessment of pulp chamber volume: a Bayesian network for the evaluation of dental evidence. International journal of legal medicine, 132(4), 1125-1138.

59. Spampinato. (2017). Deep learning for automated skeletal bone age assessment in X-ray images. Medical image analysis, 36, 41-51.

60. Srivastava, N. H., Geoffrey. (2014). Dropout: a simple way to prevent neural networks from overfitting. The journal of machine learning research, 15(1), 1929-1958.

61. Tao, J. (2019). Dental age estimation: a machine learning perspective. Paper presented at the International Conference on Advanced Machine Learning Technologies and Applications.

62. Tao, J., Chen, Mufan, Wang, Jian, Liu, Lin, Hassanien, Aboul Ella, Xiao, Kai. (2018). Dental age estimation in East Asian population with least squares regression. Paper presented at the International Conference on Advanced Machine Learning Technologies and Applications.

63. Tuan, T. M. (2017). Dental segmentation from X-ray images using semi-supervised fuzzy clustering with spatial constraints. Engineering Applications of Artificial Intelligence, 59, 186-195.

64. Van Der Maaten. (2009). Dimensionality reduction: a comparative. J Mach Learn Res, 10(6671), 13.

65. Wasule, V. S., Poonam. (2017). Classification of brain MRI using SVM and KNN classifier. Paper presented at the 2017 Third International Conference on Sensing, Signal Processing and Security (ICSSS).

66. Wik, E. H., Martínez- Silván, D., Farooq, A., Cardinale, M., Johnson, A., \& Bahr, R. (2020). Skeletal maturation and growth rates are related to bone and growth plate injuries in adolescent athletics. Scandinavian journal of medicine \& science in sports, 30(5), 894- 903. 